\title{
Applying Self-Adaptation to Automate the Management of Online Documentation of Telecom Systems *
}

\author{
Danny Weyns, IEEE Member, Morgan Ericsson, Welf Löwe, \\ Frans Frejdestedt, Johan Thornadtsson and Anna-Karin Hulth
}

\begin{abstract}
Engineering software-intensive systems, such as production systems, is complex as these systems are subject to various types of changes that are often difficult to anticipate before deployment. Tackling this complexity requires joint expertise from different backgrounds. In this paper we focus on the problem of maintaining online technical documentation of telecom systems. In the context of continuous deployment and ever-changing user needs, high quality of the documentation of such products is in a key concern of users. To tackle this problem, different experts worked together equipping the online documentation system with a feedback loop. This feedback loop tracks changes in the system and its context and automatically adapts the documentation accordingly. The results demonstrate that this self-adaptation approach offers a viable solution to tackle the maintainability problem of online documentation of telecom systems.
\end{abstract}

\section{INTRODUCTION}

Over the past decade, the engineering of softwareintensive systems has evolved towards information-driven efforts involving experts with different complementary background. This is particularly the case for production systems that involve both physical and cyber elements, often referred to as Cyber-Physical Systems. Engineering such systems requires the integration of various types of models and methods from different perspectives. To that end, modelcentric engineering approaches have been advocated that span the full lifecycle of such systems, from inception to operation and evolution [1]. In this paper we focus on the problem of maintaining the online documentation of telecom systems. In a context of continuous deployment and ever changing user needs, keeping the technical documentation in sync with the telecom products and ensuring a good structure of the documentation becomes a key concern for users (engineers, operators, etc.). Tacking this problem in an effective manner requires the joint effort of domain experts, engineers specialized in the provision of high quality product information, engineers specialized in metrics- and analyticsbased quality assessment of software and information, and experts in self-adaptation.

Central to the problem of maintaining the online documentation of telecom systems is handling change without service interruption. Change in software-intensive systems can manifest itself in different forms, ranging from strategically planned evolution of products to dynamics in the environment where the system operates and changing needs

\footnotetext{
*Support by Knowledge Foundation Sweden (Project 20150088, Software Technology for Self-Adaptive Systems). D. Weyns is with KU Leuven \& Linnaeus (e-mail: danny.weyns@kuleuven.be). M. Ericsson is with Linnaeus. W. Löwe is with Softwerk is with Linnaeus.. F. Frejdestedt is with Ericsson. J. Thornadtsson and A. Hulth are with Sigma Technology.
}

of stakeholders. Our particular focus in this paper is on the ability of systems to handle change at runtime autonomously. A prominent approach to deal with runtime change is so called self-adaptation [2]. Self-adaptation equips a software system with extra logic in the form of a feedback loop that collects data of the system and its environment that was difficult or impossible to determine before deployment [3]. The feedback loop uses the collected data to maintain runtime models. These models are then used by the feedback loop to reason about the system and to adapt it to changes in order to provide the required quality goals. An example is a self-managing client-server system that continuously tracks and analyzes changes in load and bandwidth and dynamically adapts the server configuration to provide the required quality of service to its users, while minimizing costs [4].

Over the past two decades, researchers and engineers from different fields have put extensive efforts in the understanding of how to engineer self-adaptive systems [411]. In recent years, the focus of research has been on how to provide assurances for the quality goals of self-adaptive systems that operate under uncertain operating conditions [12]. According to [11], after a relatively slow start, research in the field of self-adaptation has taken up significantly from 2006 onwards. Several self-adaptation techniques have found their way to industrial applications; a prominent example is cloud elasticity. However, the author of [11] stresses that further application of self-adaptation to practical systems will be of critical importance for the field to reach full maturity.

In this paper, we apply self-adaptation to solve the concrete problem of maintaining online technical documentation connected to telecom systems. In particular, we demonstrate how self-adaptation enabled Ericsson to automate the maintainability of online documentations of telecom systems in the context of a join effort with Sigma Technology, Softwerk, Linnaeus University, and KU Leuven. The evaluation results demonstrate that this self-adaptation approach offers a viable solution to tackle the maintainability problem of online documentation of telecom systems.

\section{SELF-ADAPTATION IN A NUTSHELL}

Researchers distinguish between two perspectives on selfadaptation: (1) the capability of a system to adapt its structure or behavior based on changes perceived in the environment, the system itself and its goals; the "self" prefix highlights that adaptation of the system is performed autonomously (i.e., with limited involvement of humans) [8], and (2) the feedback loop mechanisms that are used to realize selfadaptation, creating an explicit separation between the managed part of the system that deals with domain concerns (goals for which the system s built) and the managing part of 
the system that deals with adaptation concerns (goals about the managed part of the system). The basic building blocks of a self-adaptive system are shown in Figure 1 [11].

The environment refers to the operating context of the self-adaptive system in which the effects of the system can be observed [13]. The managed system comprises the software that realizes the domain functionality of the system. The managing system, that is responsible to manage the managed system; comprises the adaptation software that deals with a set of adaption goals. The adaptation goals refer to concerns about the managed system that are dealt with by the managing system. Adaptation goals typically relate to software qualities of the managed system; they can be subject to change themselves, which is not shown in Figure 1.

A common way to design the managing system software is by means of a Monitor-Analyzer-Planner-ExecuterKnowledge feedback loop (MAPE-K in short). The Monitor element collects data from the managed system and the environment at runtime that is used to update the Knowledge. The Analyzer uses the knowledge to determine whether the managed system needs to be adapted or not based on the adaptation goals. If an adaptation is required, the Planner puts together a plan of adaptation actions that are then executed by the Executor in order to adapt the managed system as needed.

\section{The Problem of MAINTENANCE OF ONLINE DOCUMENTATION OF TELECOMMUNICATION SYSTEMS}

\section{A. Problem Context}

Ericsson ${ }^{1}$ is a company that provides software, services, and infrastructure for communication, all of them having extensive technical documentation. An important quality of such documentation is its usability, i.e., the ability for operators and users to find the precise required information to solve a problem quickly. This requires a good structure of the technical documentation, which needs to be maintained as products and uses evolve. Sigma Technology ${ }^{2}$ is a company that is specialized in providing high quality product information for other companies, such as Ericsson. Linnaeus University has a long-term research collaboration with Ericsson and Sigma Technology to improve quality and quality assessment of technical documentation. Many of our findings are based on and incorporated in tools provided by Softwerk, ${ }^{3}$ a company specialized in metrics- and analyticsbased quality assessment of software and information.

Apart from the logical flow of the text, an important element that helps users using technical documentation is a cross-reference, i.e., a link from one document to another. Cross-references can point users to an optional step or to further details about some particular topic. However, crossreferences add dependencies between documents that can be difficult and expensive to manage when the documentation and its usage dynamically evolve. The problem is in essence similar to the maintenance of dependencies between software modules. However, mark-up languages such as HTML or XML are generally not as strict as programming languages, so even problems that a compiler would catch in the case of

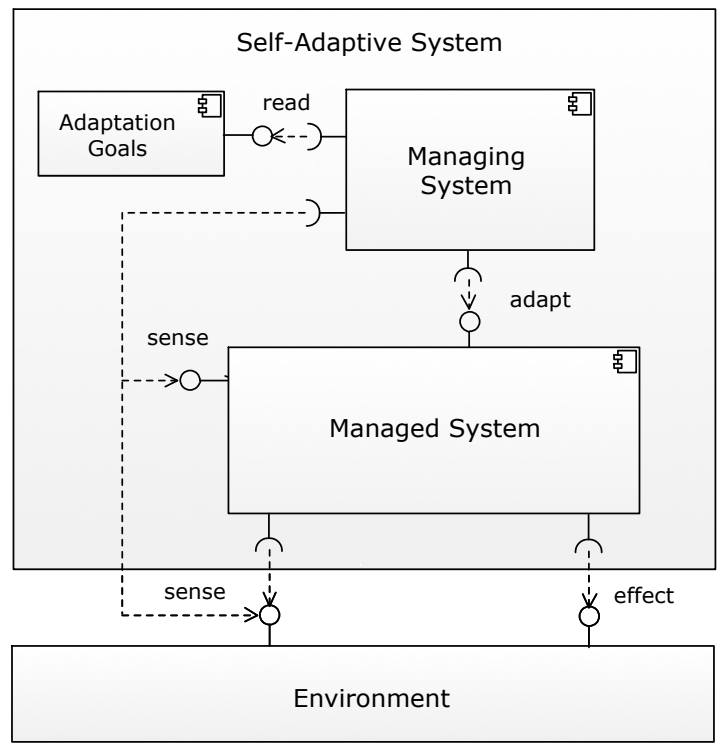

Figure 1. Basic building blocks of a self-adaptive system

software can be difficult to catch in documentation. For example, if a section was moved to a different document but the following section cross-references the section "above," this would not generate an error.

When the structure and the content of a technical documentation is checked, it is generally a manual process done by proof-readers. There are tools that can support both readers and writers, but these tools are generally not able to find or correct cross-reference and other structural problems automatically. One example of such support tools is "web analytics" tools that capture how a user interacts with the documentation. Patterns in this data can help writers to understand problems with the documentation, e.g., problems with its structure. In a joint effort between Ericsson, Sigma Technology, Softwerk, Linnaeus University and KU Leuven, we studied and developed a self-adaptive solution that employs analytics to automatically adapt the structure of a technical documentation to improve its usability and maintainability.

\section{B. Challenge}

We address the following challenge:

How can we automatically maintain a good structure of a technical documentation with high quality cross-references as products and their usages dynamically change?

Concretely, this means identifying, removing bad and unnecessary links and documents, and adding missing links.

Ericsson's technical documentation can be accessed via a web portal; this portal collects analytics data. To build a solution that tackles the challenge, we used the documentation for one of Ericsson's products and collected analytics data. The documentation consists of about 3,500 HTML documents with about 31,000 cross-references.

\footnotetext{
${ }^{1} \mathrm{https}: / / \mathrm{www}$. ericsson.com/en

${ }^{2}$ http://sigmatechnology.se/

${ }^{3}$ https://softwerk.se/
} 
The analytics data consists of a series of events grouped into user sessions. The events are either document views or searches. The data for a document view includes the document name, date and time, the time spent on the document, etc. The search data includes the search terms, whether the search resulted in a document view, and if so what document, etc. We use analytics data collected from users internal to Ericsson, so we also know their job role, organizational unit, etc. The data spans a period of about 6 months and includes about 3,200 sessions, 9,800 document views, and 5,100 successful searches.

The domain concern of the documentation system is providing users access to the online documentation. The adaptation concern is increased usability and maintainability of the telecom system documentation. The aim of adaptation is to ensure a high-quality structure of the technical documentation. The documentation should evolve while the product and the usage may change over time; these dynamics are in general difficult to predict. The quality of the documentation is determined based on cross-references. Cross-references are considered good based on how often they are used; they are considered bad when they are seldom used. A search is considered a missing cross-reference if the cross-reference actually exists but is not used; this is considered bad. The concrete adaption goals are to: (1) minimize the number of searches, (2) maximize the good cross-references, and (3) down-prioritize and eventually delete bad cross-references and unused documents.

\section{ADAPTATION SOLUTION}

We developed a first self-adaptive online documentation system that allows us to investigate how well the approach works in practice. Figure 2 shows an overview of the selfadaptive solution. The managed system consists of a web portal that offers users access to the documentation with a search engine. Figure 3 shows an excerpt of a document from the documentation we used. The documents are maintained in the document database (Figure 2). The analytics component maintains data about the documentation system and its usage. To access this data, the system offers probes that allow tracking the current structure of the documentation and the usage. In addition, the system offers effectors that can be used to restructure the documents and links, i.e., add and remove documents or cross-references.

The managing system, which is implemented in Python, is internally structured as a MAPE-K feedback loop (Figure 2). A central part of the knowledge repository is a model of the documentation represented as a directed cyclic graph. Figure 4 shows an excerpt of the graph representation. Vertices represent documents and edges represent crossreferences. Each vertex and edge has a utility probability that represents the goodness of the documentation element based on its usage, i.e., documents and cross-references. The utility probability values of document content are defined by writers of the documentation and initially set to 0.99 as a default value. The utility values of cross-references are automatically created based on a search and initially set to 0.75 . We currently only use these default values, but in the future, the values could be adjusted depending on the certainty of the usability of a cross-reference expressed by the stakeholders.

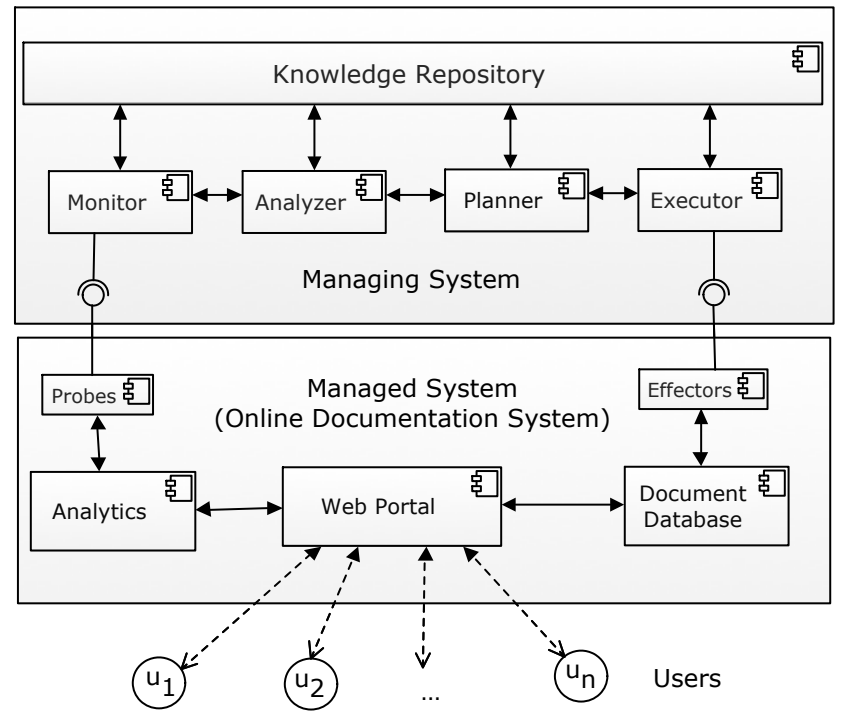

Figure 2. Architecture of self-adaptive documentation system

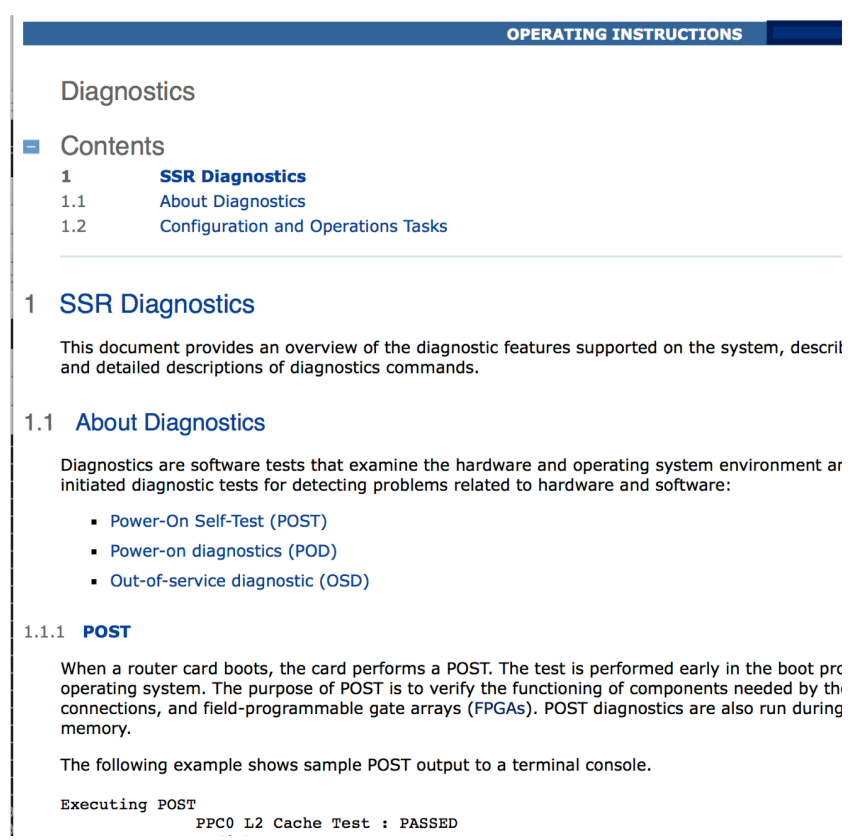

Figure 3. Excerpt of a document

The monitor component tracks the user actions captured by the analytics component. This data is used to adjust the utility probabilities of vertices and edges; this can be thought of as up- or down-voting. When a user follows a crossreference, it is up-voted, if it is not followed for a specific time period it is down-voted. To that end, we use two rules:

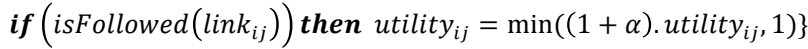

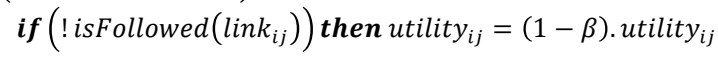

The first rule implements an up-vote. The utility probability of a link is increased with a factor $\alpha$ up to the maximum of 1 . The second rule implements a down-vote. In this case, the utility probability is decreased with a factor $\beta$. Similar rules are used for the adaptation of utility 


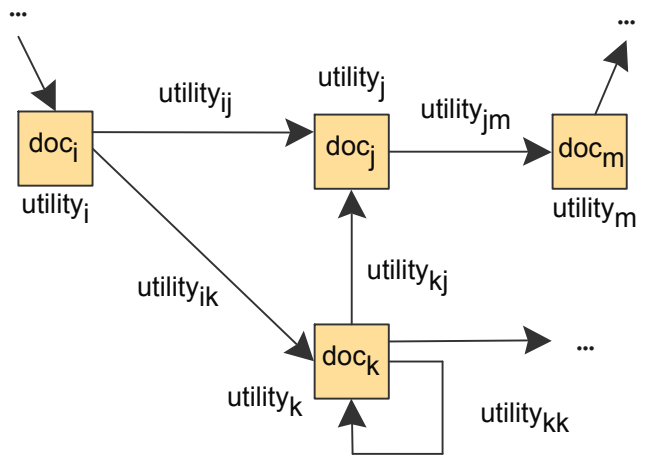

Figure 4. Excerpt of the documentation graph

probabilities of documents. In our experiments, we have set $\alpha$ to 0.2 and $\beta$ to 0.05 . These values are determined experimentally. As an example, a cross-reference that was created from a search that is respectively voted up, down, and down, would have a utility probability value of 0.81 (i.e., $0.75 \times 1.2 \times 0.95 \times 0.95 \approx 0.81)$. Recall that utility probabilities express the goodness of the link based on its usage.

The analyzer component periodically triggers analysis (e.g., every 48 hours). We use time-based triggering rather than an event-based to ensure stability. Experience shows that event-based triggering would either trigger the adaptation too often or delay changes for too long. If adaptation is triggered too often, cross-references may be removed prematurely even if their usability probability is still high (since they are checked so often). The task of the analyzer consists of two parts. First, it analyzes the documentation graph and identifies the documents and crossreferences that require adaptation. To that end, the analyzer walks through the documentation graph and checks the utility probability for each documentation element to determine whether to keep the element or to remove it. For links this is simply realized as follows:

$$
\begin{aligned}
& \text { if }\left(\text { lastAccess }\left(\text { link }_{i j}\right)+\text { threshold }<\text { currentTime }\right) \text { then }\{ \\
& \text { if }\left(\operatorname{random}(0,1)>\text { utility }_{i j}\right) \text { then remove }\left(\operatorname{link}_{i j}\right) \\
& \text { else } \left.\text { utility }_{i j}=(1-\beta) \text {. utility }{ }_{i j}\right\}
\end{aligned}
$$

A link can only be removed if it has not been accessed over a given threshold period (e.g. 7 days). A similar rule is defined for documents. If an element should not be kept, it is annotated with a remove mark in the documentation graph. Second, the analyzer identifies the searches that have happened since the previous adaptation cycle. In particular, searches where the user spends more than a particular time window viewing a target document (e.g. 2 minutes). For each such a search, a linksource is added to the documentation graph at the document that the user viewed when the search was initiated and an linktarget is added to the document that was viewed for the given time window.

The planner then creates the set of adaptation steps needed to adapt the documentation. For each documentation element marked with a remove mark an adaptation action is added to the plan to remove the element. In case a document needs to be removed, existing links to this document are also removed. For each pair of linksource and linktarget an adaptation action is added to the plan to create the new crossreference in the documentation.

\section{EVALUATION}

To evaluate the self-managing documentation system, we used a proof-of-concept system with analytics data that we collected from Ericsson's web portal. Recall that the documentation consists of 3,500 documents that are connected via about 31,000 cross-references. The experiment spans over a period of 6 months with about 3,200 sessions, 9,800 document views, and 5,100 successful searches. Adaptations of the documentation are performed based on usage and analytics are adjusted based on the adaptations. For instance, if a search triggers the creation of a cross-reference, future identical searches reduce the utility that cross-reference generated from the search.

The system is evaluated based on the miss ratio, i.e., the number of times a user had to search for something that has been removed prematurely. We also evaluate the solution based on how many cross-references are removed and when. A cross-reference should exist as long as it is in use.

In total, we applied 80 adaptation cycles in the 6 months period, with 48-hour adaptation periods.

Figure 4 gives an overview of the results. The adaptation process consists of two phases: and initial optimization phase followed by the regular adaptation phase. The first 25 adaptation cycles the initial documentation goes through a rapid optimization process where unused cross-references are removed. Concretely, all cross-references are initially given a 7-day grace period before they can be removed, so over the first few adaptation cycles, the documentation can only grow due to newly introduced cross-references. At cycle 4 , the grace period is over, and until cycle 25 most of the crossreferences that have not been used are removed.

After the initial optimization phase the system enters the adaptation phase. The system evolves towards a working set of documents with cross-references that are dynamically adjusted and maintained. Figure 5 show a detailed view on the adaptation phase from cycle 30 to 80 . Besides the change in the number of cross-references, the figure also shows the number of misses (per cycle). From cycle 30 till cycle 36, the documentation remains relatively stable. From cycle 36 to cycle 76 , we observe a further reduction of the number of cross-references. We inspected the analytics data in this

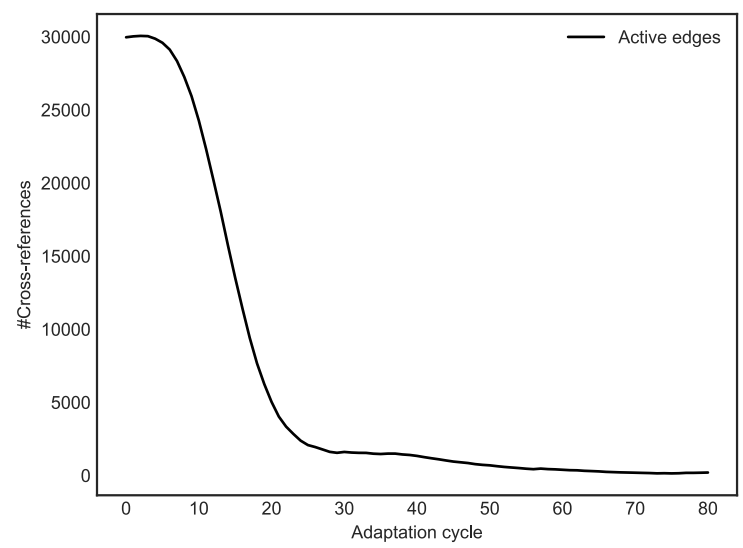

Figure 4. Number of cross-references over 80 adaptation cycles 
period and observed a reduction in the number of searches. The self-adaptive system responds with reducing the size of the working set. Further inspection shows that the reduction was caused by a new release of the documentation around cycle 36 marked with (1), so many users move to the newer release. Around cycle 76 marked with (2), we observe an increase of searches and misses. This disturbance is caused by another update of the documentation; the data shows that the documentation lacks links to support the users; so new cross-references are dynamically created and added.

The total number of misses over the 6 months is 2,817 , or about $29 \%$. In order to not count once-in-a-lifetime searches as misses, we remove all corresponding misses, of which there are 2,075. The adjusted miss rate is about $8 \%$. The average time from adding a reference to deletion is about 32 days (or 16 adaptation cycles).

On average it takes 17 adaptation cycles (worst case 37 cycles) to remove links that are not used (at that time, the estimated goodness is on average about $40 \%$ ). Based on our goal to (1) remove links that are neither required nor useful, (2) reduce the overall number of misses, and (3) if a miss happens, the time between last use and the miss should be large, the self-adaptive system behaves as expected.

To obtain further insight in the adaptation behavior of the self-adaptive system, we created two types of simulated users derived from historical data. The behavior of the users can be controlled using parameters; e.g., the number of visited documents, the probability of a search, etc. One type of users works with a limited working set of 300 documents (representing experts); the other type browses the full range of documents (representing inexperienced users). We randomly generated different behaviors and different user populations. The simulations span on average 18 months (about 270 adaption cycles) and contain about 1,000,000 document views. Figure 6 shows the adaptations of crossreferences over time. The results with simulated users correspond with those from the sessions with real users. After an initial optimization phase, the working set converges and is dynamically adapted. Unused documents are removed from the system and the number of cross-references dynamically adapts with document updates and searches.

\section{INSIGHTS AND LESSONS LEARNED}

To get further insights in the opinions on the self-adaptive solution, we performed semi-structured interviews with the technical staff of Ericsson, Sigma Technology, and Softwerk. We summarize the main outcome on four guiding questions.

Q1: What do you consider the most important benefits of the self-adaptive solution?

The staff emphasised that it is difficult to (statically) determine whether cross-references are of high quality and if they are needed or not. Traditionally, analytics are collected and used by technical writers and proof-readers to get an idea which cross-references are used, how they are used, when search is required, etc. However, the analytics are only an indication. A major benefit of the self-adaptive solution is that it uses the analytics to automatically determine whether cross-references are successful or not, resulting in more costeffective creation of higher-quality documentation.

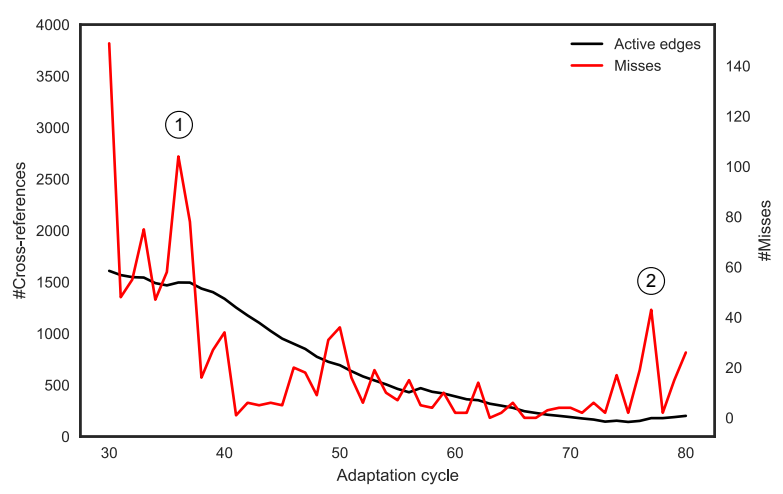

Figure 5. Cross-references and misses during adaptation phase

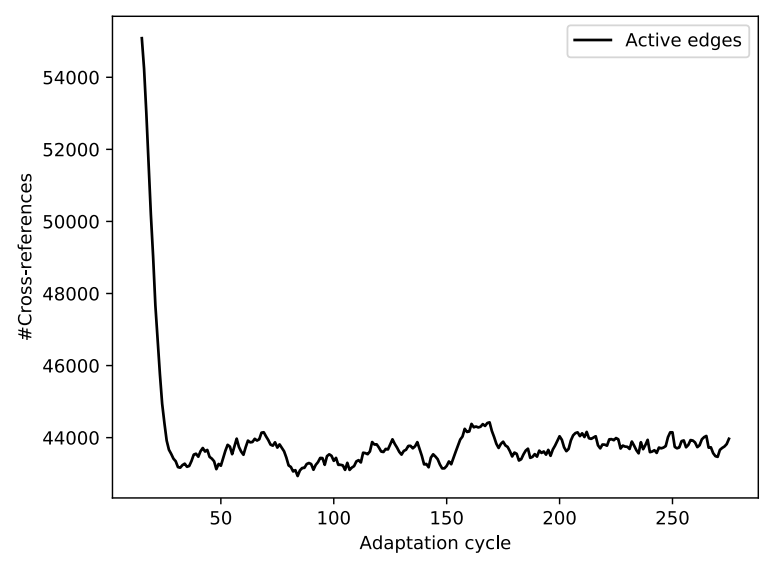

Figure 6. Adaptation results with simulated users

Q2: What are potential risks of the self-adaptive solution and how could these risks be mitigated?

The main risks are related to the quality of the documentation. If the self-adaptive solution made a series of bad decisions, it would possibly have a big impact on the usefulness of the documentation for the end users. The current focus has been on managing cross-references, in part because they directly impact quality, but also because bad decisions can be mitigated relatively easily. In particular, an end user can always use search, index, or table of contents to find the right information. Another idea to mitigate this risk could be to enable human experts to perform intermediate quality checks, which could help increasing user trust. Another potential risk is that there is not enough analytics data available. For example, some documentation is tied to specific releases of a product, and when newer releases are available, the older documentation is less frequently used. This risk could be mitigated by making the self-adaptive solution not only aware of the usage patterns, but also of the frequency of use. Finally, integrating automatically generated cross-references in a natural manner may be difficult. Improving rewrite tools and templates can mitigate this risk.

Q3: Do you see a broader applicability of the proposed solution for your business? 
Parts of technical documentation, others than crossreferences, could also be adapted. Most of the current documentation is written in narrative form (monolithic documents), but as we move to topic-oriented documentations, where each unit (e.g., page or document) of the documentation is focused on a specific topic, the entire documentation could be adapted. For example, if a series of topics are frequently accessed together, they could be joined to form a larger super-topic. Further study is required to see whether the proposed method can be extended to cover a broader range and variety of products.

\section{Q4: Do you have any particular lessons learned?}

One important lesson learned by the partners is that selfadaptive systems provide a competitive advantage in maintenance tasks today when compared to human efforts. While further experience needs to be obtained for rolling out automated management at large scale, the Ericsson staff considers integrating self-adaption as a standard feature in the documentation system as a particularly promising direction.

Another key lesson that the team learned from the joint effort (and previous joint efforts) is that many engineering techniques that are developed for software are also applicable to technical documentation connected to software-intensive systems. Examples are metrics, quality models, architecture, and analysis techniques. In most cases, the differences are in the details; e.g., the specific metrics and models that are used to measure quality. Sometimes applying a technique can be trivial in one area but difficult in the other one. For example, in a context of software knowing whether a class contains a method that is referenced can often simply be checked by a compiler. Answering a similar question for technical documentation is significantly more difficult as it requires encoding and resolving the semantics of references. On the other hand, collecting analytics and runtime information about the users is almost trivial since we generally have full access to both the server and the reading platform.

\section{RELATED EFFORTS}

Various leading ICT companies have invested significantly in the study and application of self-adaptation techniques [14][15], including initiatives such as IBM's Autonomic Computing, Sun's N1, HP's Adaptive Enterprise, and Microsoft's Dynamic Systems. These efforts have resulted in industrial applications, such as automated server management, cloud elasticity and automated data center management. ${ }^{4}$ These efforts primarily target automatic management of resources and infrastructure of computing systems. A number of recent R\&D efforts have explored the application of self-adaptation beyond mere resource and infrastructure management. For example, [16] applies self-adaptation to an industrial middleware to monitor and manage highly populated networks of devices, while [17] applied self-adaptive techniques to role-based access control for business processes. Our work contributes to these efforts with the application of self-adaptation in

4 kief.com/automated-server-management-lifecycle.html; aws.amazon.com/ec2/; $\underline{\text { device42.com/solutions/automated-data-center-management }}$ practice in the domain of automatic maintenance of online documentations of software-intensive systems.

\section{CONCLUSION}

In this paper we realized a self-adaptation solution to an engineering problem of a software-intensive system by combining different expertise. The results show that selfadaptation offers a viable solution for solving this concrete problem in practice. The partners recognize that the selfadaptive solution increases the qualities of their products and reduces costs. Nevertheless, there is no free lunch. Selfadaptation comes with some risks. One important risk is uncertainty whether the self-adaptive solution may not make a series of bad decisions, which may jeopardize the quality of the documentation. Another one is uncertainty about whether sufficient analytics data will always be available to make proper adaptation decisions. These risks can be mitigated, for example by enriching the information collected by the feedback loop when necessary. On the other hand, these risks provide the basis for further research and collaboration between the partners involved in this effort.

\section{REFERENCES}

[1] D. Garlan, Modeling Challenges for Cyber-Physical Systems, Software Engineering for Smart Cyber-Physical Systems, SEsCPS'15

[2] P. Oreizy et al., An architecture-based approach to self-adaptive software, IEEE Intelligent Systems and Applications, 14(3), 1999.

[3] J. Kephart and D. Chess, The Vision of Autonomic Computing, Computer 36(1), 2003.

[4] D. Garla et al., Rainbow: Architecture-Based Self-Adaptation with Reusable Infrastructure, Computer 37(10), 2004

[5] S. Dobson et al., A. survey of autonomic communications, ACM Transactions on Autonomous and Adaptive Systems, 1 (2), 2006

[6] J. Kramer and J. Magee, Self-Adaptive Systems, An Architecture Challenge, Future of Software Engineering, FOSE 2007

[7] M. Salehie and L. Tahvildari. Self-adaptive software: Landscape and research challenges. ACM TAAS Systems 4(2), 2009.

[8] B. Cheng et al., Software Engineering for Self-Adaptive Systems: A Research Roadmap, Software Engineering for Self-Adaptive Systems, Lecture Notes In Computer Science, Vol. 5525. Springer, 2009.

[9] D. Weyns, S. Malek, and J. Andersson, FORMS: Unifying reference model for formal specification of distributed self-adaptive systems. ACM Transactions on Autonomous and Adaptive Systems 7(1), 2012.

[10] R. de Lemos et al., Software Engineering for Self-Adaptive Systems: A Second Research Roadmap, LNCS Vol. 7475. Springer 2013.

[11] D. Weyns, Software Engineering of Self-Adaptive Systems: An Organized Tour and Future Challenges, Handbook of Software Engineering, Springer 2018.

[12] R. de Lemos et al., Software Engineering for Self-adaptive Systems: Research Challenges in the Provision of Assurances, Software Engineering for Self-Adaptive Systems III, LNCS Springer, 2017.

[13] M. Jackson, The Meaning of Requirements, Annals of Software Engineering 3, 1997

[14] Y. Brun, Improving Impact of Self-adaptation and Self-management Research Through Evaluation Methodology. In Software Engineering for Adaptive and Self-Managing Systems, 2010

[15] H. Muccini, M. Sharaf, and D. Weyns. Self-adaptation for cyberphysical systems: a systematic literature review, SEAMS, 2016

[16] J. Cámara et al., Evolving an Adaptive Industrial Software System to Use Architecture-based Self-Adaptation, Software Engineering for Adaptive and Self-Managing Systems, 2013

[17] C. E. Da Silva et al., Self-Adaptive Role-Based Access Control for Business Processes, SEAMS, 2017 